\title{
The long non-coding RNA NONHSAG026900 predicts prognosis as a favorable biomarker in patients with diffuse large B-cell lymphoma
}

\author{
Shuangtao Zhao ${ }^{1, *}$, Shuangsang Fang ${ }^{2, *}$, Yanhua Liu ${ }^{3,4,5, *}$, Xixi Li ${ }^{3,6}$, Shengyou Liao ${ }^{2}$, \\ Jinwen Chen ${ }^{2}$, Jingjia Liu ${ }^{2}$, Lianhe Zhao ${ }^{2}$, Hui Li $^{2}$, Wei Zhou ${ }^{3,4,5}$, Wenzhi Shen ${ }^{3,4,5}$, \\ Xiaoli Dong ${ }^{3,4,5}$, Rong Xiang ${ }^{3,4,5}$, Luhua Wang ${ }^{1}$, Yi Zhao ${ }^{2}$ \\ ${ }^{1}$ Department of Radiation Oncology, National Cancer Center/Cancer Hospital, Peking Union Medical College and Chinese \\ Academy of Medical Sciences, Beijing, China \\ ${ }^{2}$ The Key Laboratory of Intelligent Information Processing, Institute of Computing Technology, Chinese Academy of Sciences, \\ Beijing, China \\ ${ }^{3}$ The School of Medicine, Nankai University, Tianjin, China \\ ${ }^{4}$ The Collaborative Innovation Center for Biotherapy, Nankai University, Tianjin, China \\ ${ }^{5}$ The Tianjin Key Laboratory of Tumor Microenvironment and Neurovascular Regulation, Tianjin, China \\ ${ }^{6}$ Department of Pathology, Nankai University, Tianjin, China \\ * Co-first author \\ Correspondence to: Rong Xiang, email: rxiang@nankai.edu.cn \\ Luhua Wang, email: wlhwq@yahoo.com \\ Yi Zhao, email: biozy@ict.ac.cn
}

Keywords: diffuse large B-cell lymphoma, long non-coding RNA, NONHSAG026900, prognosis, biomarker

Received: January 17, 2017 Accepted: February 24, $2017 \quad$ Published: March 13, 2017

Copyright: Zhao et al. This is an open-access article distributed under the terms of the Creative Commons Attribution License (CC-BY), which permits unrestricted use, distribution, and reproduction in any medium, provided the original author and source are credited.

\section{ABSTRACT}

Long non-coding RNAs are known to be involved in cancer progression, but their biological functions and prognostic values are still largely unexplored in diffuse large B-cell lymphoma. In this study, long non-coding RNAs expression was characterized in 1,403 samples including normal and diffuse large B-cell lymphoma by repurposing 7 microarray datasets. Compared with any stage of normal B cells, NONHSAG026900 expression was significantly decreased in tumor samples. And in germinal center B-cell subtype, the significantly higher expression of NONHSAG026900 indicated it was a favorable prognosis biomarker. Then the prognostic power of NONHSAG026900 was validated with another independent dataset and NONHSAG026900 improved the predictive power of International Prognostic Index as an independent factor. Moreover, functional prediction and validation demonstrated that NONHSAG026900 could inhibit cell cycle activity to restrain tumor proliferation. These findings identified NONHSAG026900 as a novel prognostic biomarker and offered a new therapeutic target for diffuse large B-cell lymphoma patients.

\section{INTRODUCTION}

Diffuse large B-cell lymphoma (DLBCL) is biologically heterogeneous and accounts for $30-35 \%$ [1] of all non-Hodgkin lymphomas (NHLs). The standard chemotherapy regimen of CHOP (cyclophosphamide, doxorubicin, vincristine, and prednisone), especially R-CHOP (rituximab, cyclophosphamide, doxorubicin, vincristine, and prednisone), has significantly improved the survival of patients with DLBCL $[2,3]$. However, approximately one-third of patients will relapse shortly after initial remission and eventually succumb to this refractory disease [4-6]. In clinical practice, the International Prognostic Index (IPI) does not fully represent DLBCL heterogeneity, despite its status as one of the most important clinical prognosis predictors [7]. Therefore, some novel prognostic factors are being explored to help predict treatment outcome.

Long non-coding RNAs (lncRNAs) are defined as non-protein-coding RNAs of more than 200 nucleotides in length [8]. Although lacking protein coding capability, IncRNAs were reported as biomarkers for predicting prognosis, metastasis, and in multiple disease diagnosis [9-12]. It is possible to detect the quantity of lncRNAs because of the relative stability of their secondary structures in the body [13]. Many lncRNAs are reported to be important in regulating cancer cell proliferation, invasion, and metastasis $[14,15]$. Peng et al. reported that 
lincRNA-p21 could predict favorable clinical outcome and impair tumorigenesis in DLBCL patients with an R-CHOP regimen [16]. However, the biological functions and prognostic value of IncRNAs in DLBCL are still largely unexplored.

In our study, we investigated lncRNA functions in 7 GEO databases, including five differentiated stages of normal B cells (naive B cells, centroblasts, centrocytes, memory B cells, and plasma cells) and DLBCL samples. Our research identified that NONHSAG026900 was significantly down-regulated in DLBCL samples, and could serve as a favorable biomarker to predict prognosis of DLBCL patients. Additionally, we integrated proteincoding gene expression into a co-expression network to predict the possible function of NONHSAG026900.

\section{RESULTS}

\section{Transcription analysis and the differential regulation of IncRNAs between normal and DLBCL samples}

Utilizing the published GEO dataset GSE12453 we concentrated on repurposing the data, including 11 DLBCL samples and 25 normal B cells samples represented five differentiated stages (naive B cells, centroblasts, centrocytes, memory B cells and plasma cells). We applied ncFANs software to re-annotate all the collected probes in the Affymetrix microarray platforms (HGU 133 plus 2.0). The probes were stratified into two groups comprising 14,707 protein-coding genes and 6,307 lncRNAs in GSE12453. We then reanalyzed the expression profiles between normal samples and DLBCL samples. As a result, we obtained 226 proteincoding genes (Supplementary Table 1) and 14 lncRNA genes (Figure 1A) with significantly different expressions $(P<0.05$, and Fold change $>2)$.

Because DLBCL might occur at any stage of normal B cell development, we compared the 14 lncRNAs expression among the five stages of normal B cell differentiation and the DLBCL stage with one-way ANOVA (with Bonferroni correction). Thus, we found that NONHSAG026900 was the common significantly alterative $\operatorname{lncRNA}(P=0.000$, Figure 1B) and it was downregulated significantly in DLBCL samples $(P=0.000$, Figure 1C).

To confirm our findings, we validated the expression profile of NONHSAG026900 in another data set GSE56315, which consisted of 33 normal samples with five different stages and 74 DLBCL samples. Consistent with the result above, NONHSAG026900 was significantly lower expression in DLBCL samples than the other normal groups $(P=0.000$, Figure 1C). These results demonstrated that a decreased expression of NONHSAG026900 might be associated with the presence of DLBCL.

\section{Potential NONHSAG026900 transcriptional control mechanism}

To explore the mechanism of much lower expression of NONHSAG026900 in DLBCL than normal, we identified a CpG methylation site 146 bp upstream of the transcription start site for NONHSAG026900 in 8 cell lines (GM12891, GM12892, GM06990, HeLa-S3, HCT-116, NB4, BJ, and U87; Figure 1D) by using the ENCODE database. The data revealed that there were no methylated sites in normal B lymphocyte cell lines (GM12891, GM12892, and GM06990), whereas partially or completely methylated sites were found in human B-cell lymphoma cell lines (BJ) and four other cancer cell lines: a cervical cancer cell line (HeLa-S3), a colon cancer cell line (HCT-116), a leukemia cell line (NB4), and a brain star glioblastoma cell line (U87). Because a promoter with DNA methylation could suppress the expression of the gene under the control of that promoter [17], this result could partially explain the low levels of NONHSAG026900 expression in tumor tissues. However, these discoveries should be validated by experimentation.

\section{Identification of diagnostic power from the NONHSAG026900 value distribution}

To further explore the expression patterns of NONHSAG026900, we performed a deeper analysis of its distribution between GCB and non-GCB subtypes in 51 patients with DLBCL from GSE56315. Our analysis indicated that patients with GCB subtype had significantly higher values of NONHSAG026900 than non-GCB subtype ( $P=0.000$, Figure $2 \mathrm{~A})$. Similarly, we confirmed the result above in GSE11318 $(n=170, P=0.000$, Figure 2B). So, we concluded NONHSAG026900 might have diagnostic potential deduced by its distribution status between GCB and non-GCB subgroups. Also, because the GCB-DLBCL patients were reported to have a more favorable outcome than those with non-GCB subtype [18], we inferred that NONHSAG026900 could act as a favorable biomarker of prognosis in DLBCL patients.

\section{Identification of prognostic power from the NONHSAG026900 value distribution}

To gain further insights into the prognostic role of NONHSAG026900 in DLBCL we analyzed the association between the NONHSAG026900 expression and clinical features in 170 patients treated with the CHOP regimen from GSE11318. According to the mean value of NONHSAG026900 expression in patients, we classified them into two groups: a low value group $(<4.96$, $n=116)$ and a high value group $(\geq 4.96, n=54$; Figure 3 and Table 1). We discovered that NONHSAG026900 was not correlated with gender $(P=0.867)$, age $(P=0.055)$, Ann Arbor stages $(P=0.980)$, ECOG performance 
status $(P=0.889)$, LDH ratio $(P=0.124)$, extra nodal sites $(P=0.996)$, or IPI score $(P=0.350)$. However, NONHSAG026900 expression was closely correlated with GCB vs. non-GCB genotype $(P=0.000$, Table 1$)$.

Next, we performed log-rank tests between the low and high value groups in GSE11318. We found that patients with low value expressions of NONHSAG026900 had higher risk than those with high values (hazard ratio $(\mathrm{HR})=1.716,95 \% \mathrm{CI}: 1.144-2.574)$, and that 5-year overall survival (OS) rates $(38.67 \%)$ in the group with low-NONHSAG026900 were significantly poorer than those $(58.82 \%)$ in the high-NONHSAG026900 group $(P=0.009$, Figure 4A). Generally, these results suggested that NONHSAG026900 could be used as a favorable biomarker associated with prognosis in DLBCL patients.

\section{Validation of NONHSAG026900 for survival prediction in GEO data sets}

To confirm our discoveries, we selected another four GEO datasets to validate the prognostic power of NONHSAG026900. Similarly, we stratified patients of each independent cohort into two groups (low and high value) by using the mean values as the cutoff point. In accordance with the results above from patients treated with a CHOP regimen in GSE11318, patients with a CHOP regimen from GSE10846 in the low value group $(n=109)$ had a higher risk $(\mathrm{HR}=1.926,95 \% \mathrm{CI}: 1.306-2.840)$ than those in the high value group $(n=72)$, while 5-year OS rates $(37.28 \%)$ in the low value group were significantly poorer than those $(61.16 \%)$ in the high value group $(P=$ 0.0009 , Figure 4B). Mean value-based classification of another cohort with a CHOP regimen from GSE53786 $(n=45)$ also produced similar results (Figure 4B). The HR (low vs. high group) in this cohort was 1.860 (95\% CI: $0.830-4.168)$. In addition, 5-year OS rates in the low value group (41.92\% vs. $60.00 \%)$ were significantly worse compared with the high value group in GSE53786 $(P=0.0132$, Figure 4B). All these results suggested that NONHSAG026900 could predict survival of DLBCL patients treated with a CHOP regimen, and function as a favorable biomarker.

To validate the prognostic power of NONHSAG026900 in patients with an R-CHOP regimen, four cohorts (GSE10846, $n=233$; GSE53786, $n=71$; GSE23501, $n=64$; and GSE31312, $n=484$ ) were included in our study. In these independent subsets of patients who were treated with an R-CHOP regimen we confirmed the reliability of NONHSAG026900 in predicting survival. The mean value classification stratified each group of patients into two subgroups (low and high value groups) with significantly different HRs $(\mathrm{HR}>1.5, P<0.05$,
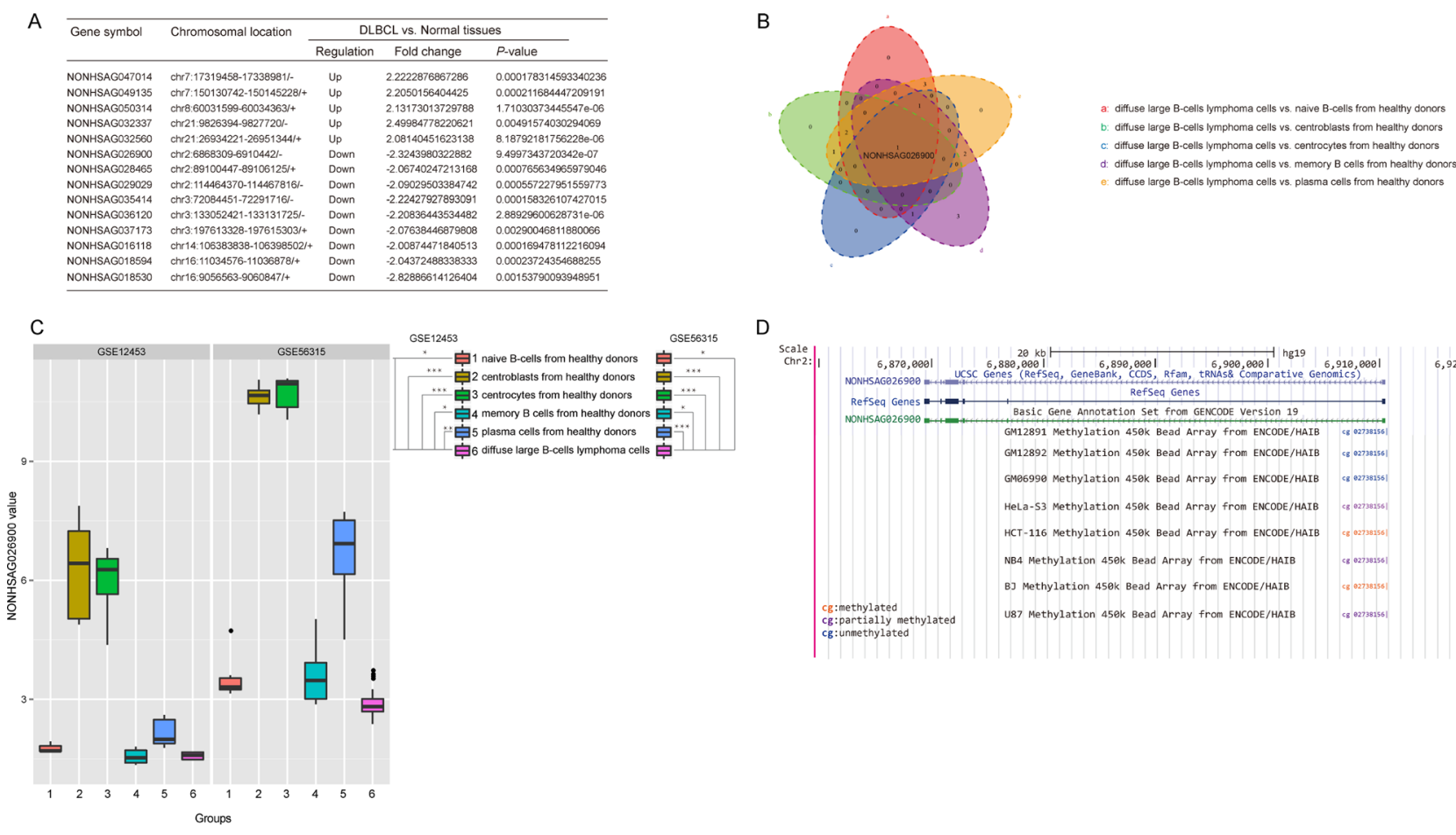

D

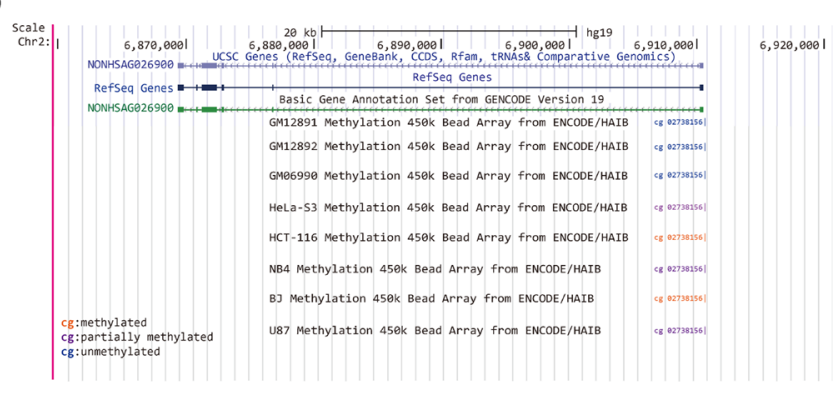

Figure 1: Screening the significant IncRNAs that were differentially expressed between normal and DLBCL tissues. (A) A total of 5 lncRNAs were up-regulated, while 9 lncRNAs were down-regulated, in DLBCL compared with normal tissues (Fold change $>2.0, P<0.05, t$-test method). (B) A Venn diagram revealed the overlapping genes among the five groups which were composed of the significantly different genes between DLBCL cells and normal B cells in each differentiation step. $P$ values were calculated with a one-way ANOVA method (Bonferroni correction). (C) Boxplots revealed that the expression of NONHSAG02900 was significantly downregulated in DLBCL compared with normal tissues in GSE12453 and GSE56315 ( $P<0.05$, one-way ANOVA method). (D) Genomic context of NONHSAG026900. A CpG methylation site was discovered upstream of the NONHSAG026900 coding sequence. 
Figure 4C). Also, patients with low expression values had significantly shorter 5-year OS rates $(63.29 \%$ vs. $89.15 \%$ in GSE10846, $P=0.0062 ; 65.45 \%$ vs. $91.67 \%$ in GSE53786, $P=0.0064 ; 72.68 \%$ vs. $94.74 \%$ in GSE23501, $P=0.013$; and $56.97 \%$ vs. $69.68 \%$ in GSE31312, $P=0.007$; Figure 4C) or progression-free survival (PFS) rates $(65.24 \%$ vs. $85.26 \%$ in GSE23501, $P=0.014$; and $43.48 \%$ vs. $61.89 \%$ in GSE31312, $P=0.003$; Figure 4D) than those with high value expression in the four validated cohorts.

Next, we combined the two groups of patients with CHOP or R-CHOP regimens into one cohort in GSE10846

A

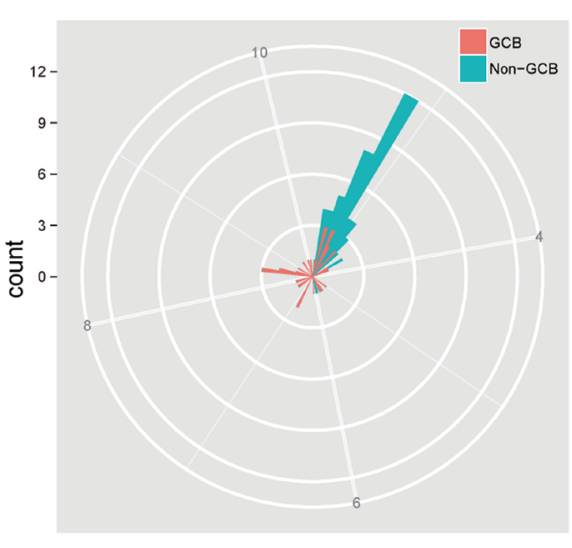

NONHSAG026900

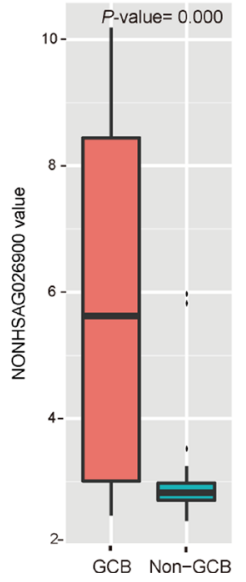

$(n=420)$ or GSE53786 $(n=117)$ to verify the predictive power of NONHSAG026900 in clinical outcomes. We obtained a similar result with patients with single CHOP or $\mathrm{R}-\mathrm{CHOP}$ regimens. Patients in the low value group were at higher risk than those in the high value group $(\mathrm{HR}=2.005$ (95\% CI:1.452-2.768) in GSE10846; and HR = 2.039 (95\% CI:1.065-3.901) in GSE53786). 5-year OS rates (46.00\% in GSE10846 and 52.91\% in GSE53786) in the low value group were significantly worse than those in the high value group $(70.46 \%$ and $73.77 \%$ in GSE10846 and GSE53786, respectively; Figure 4E). Generally, NONHSAG026900 could predict the outcome of
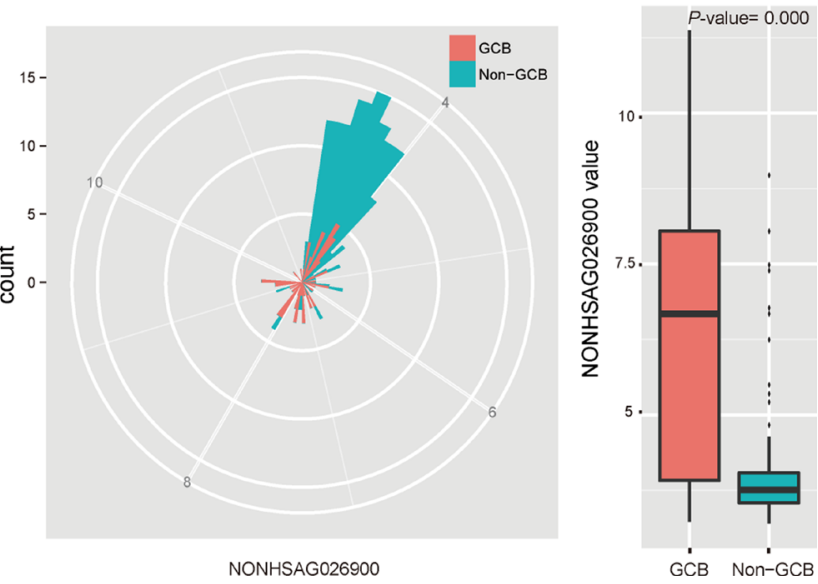

Figure 2: Analysis of the predictive power of NONHSAG026900 from the distribution of its expression values. (A) The radar map method and boxplot showed that the value of NONHSAG026900 was significantly greater in the GCB than non-GCB subgroup in GSE56315 ( $P=0.000, t$-test method). (B) The radar map method was used for validating that the distribution of NONHSAG026900 expression values between GCB and non-GCB subgroups in GSE11318 was similar to that in GSE56315, while the boxplot analysis demonstrated that the value of NONHSAG026900 was significantly lower in the non-GCB subtype DLCBL patients in GSE11318 $(P=0.000, t$-test method).

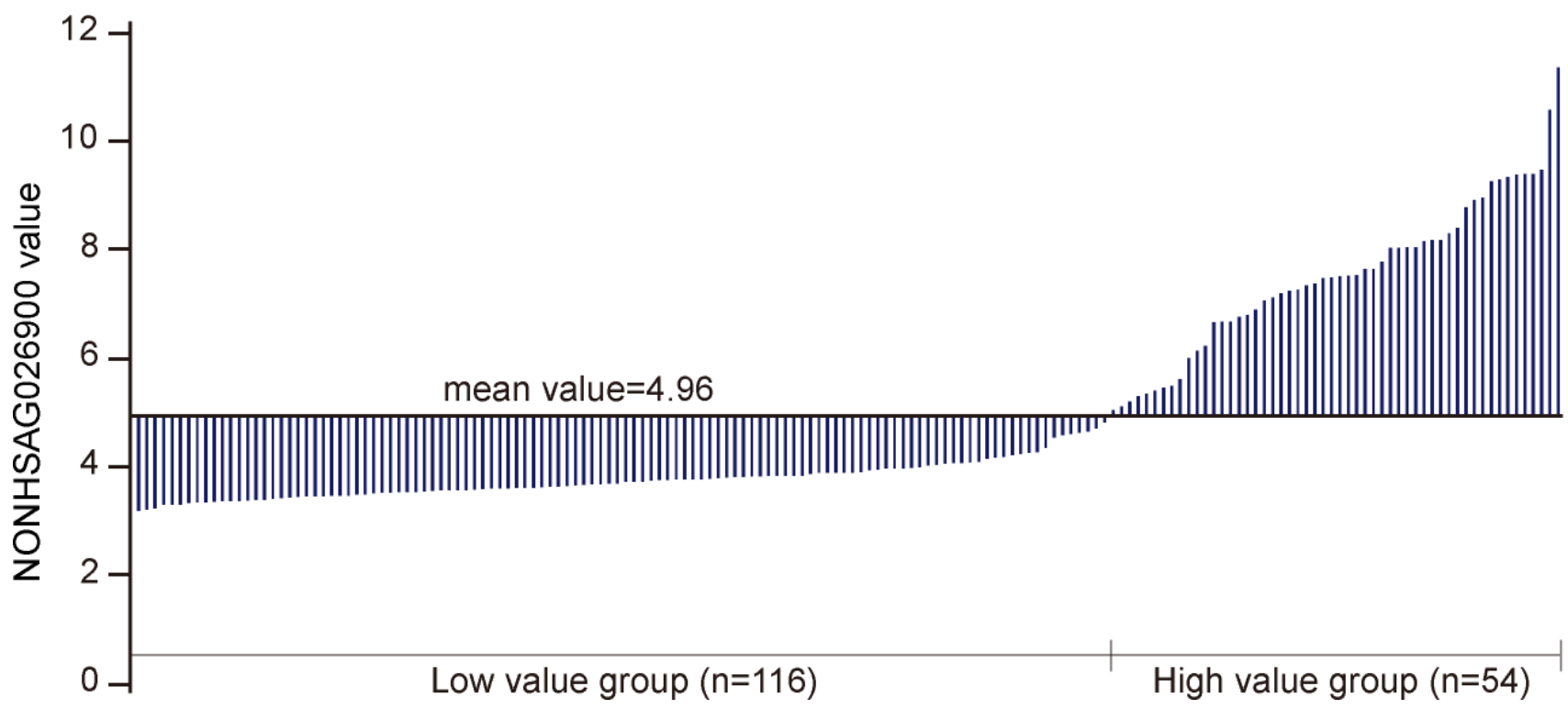

Figure 3: Divide the NONHSAG026900 IncRNA levels into two groups. Stratification of the 170 DLBCL patients from GSE11318 into two groups (low value group, $n=116$ and high value group $n=54$ ) via the mean value of NONHSAG026900 expression. 
Table 1: Correlation between NONHSAG026900 and clinicopathological characteristics in DLBCL

\begin{tabular}{|c|c|c|c|c|}
\hline \multirow{2}{*}{ Characteristics } & \multirow{2}{*}{$n$} & \multicolumn{2}{|c|}{ NONHSAG026900 expression } & \multirow{2}{*}{$P$-value } \\
\hline & & Low value & High value & \\
\hline Gender & & & & 0.867 \\
\hline Male & 96 & 65 & 31 & \\
\hline Female & 74 & 51 & 23 & \\
\hline Age (years) & & & & 0.055 \\
\hline$<60$ & 64 & 38 & 26 & \\
\hline$\geq 60$ & 99 & 73 & 26 & \\
\hline Ann Arbor stages & & & & 0.980 \\
\hline I-II & 75 & 51 & 24 & \\
\hline III-IV & 87 & 59 & 28 & \\
\hline ECOG performance status & & & & 0.889 \\
\hline $0-1$ & 122 & 83 & 39 & \\
\hline $2-4$ & 39 & 27 & 12 & \\
\hline LDH ratio & & & & 0.124 \\
\hline$\leq 1$ & 68 & 40 & 28 & \\
\hline$>1$ & 76 & 54 & 22 & \\
\hline Extra nodal sites & & & & 0.996 \\
\hline$<2$ & 134 & 91 & 43 & \\
\hline$\geq 2$ & 28 & 19 & 9 & \\
\hline IPI score & & & & 0.350 \\
\hline $0-2$ & 104 & 66 & 38 & \\
\hline $3-5$ & 39 & 28 & 11 & \\
\hline Genotype & & & & $0.000 *$ \\
\hline $\mathrm{GCB}$ & 70 & 26 & 44 & \\
\hline Non-GCB & 100 & 90 & 10 & \\
\hline
\end{tabular}

*Statistically significant $(P<0.05)$, Pearson Chi-Square test.

DLBCL as a favorable biomarker, no matter what kind of treatments (CHOP or R-CHOP) were selected for patients in clinical practice.

Table 2 revealed that the NONHSAG026900 expression value was significantly associated with OS as a continuous variable in GSE11318 and the four validation cohorts $(P<0.05)$ using a univariate Cox regression analysis. The association of the NONHSAG026900 expression value and OS was also significant $(P<0.05$, Table 2) when it was measured as a continuous variable in a multivariate analysis with Cox regression including another three prognostic factors including gender, genotype and IPI score (composed of age, Ann Arbor stage, ECOG performance status, LDH ratio, and extra nodal sites). These results confirmed the ability of the NONHSAG026900 in predicting survival as an independent factor.

\section{NONHSAG026900 is superior to other biomarkers and adds to the predictive power of the IPI}

Multivariate Cox regression showed that NONHSAG026900 expression levels acted as an independent prognostic factor in DLBCL patients. A ROC curve was produced to compare the prognostic power of NONHSAG026900 expression to 30 prognostic biomarkers reported publicly [19-24] in 170 DLBCL patients from GSE11318. The area under the ROC curve
(AUC) was $0.703(P=0.003$, Figure 5A), which indicated that the NONHSAG026900 had more diagnostic power for DLBCL than these other 30 biomarkers. Unfortunately, the predictive power of NONHSAG026900 was slightly inferior to the IPI (AUC $=0.715, P=0.000$; Figure $5 \mathrm{~B}$ ).

Next, we investigated whether NONHSAG026900 could add to the predictive power of the IPI. We divided patients into two groups (low IPI: 0-2 and high IPI: 3-5) based on the IPI scores. Because there were too few (only 39) patients in the high IPI group from GSE11318 to achieve statistical significance, we selected GSE10846 as an analysis dataset to perform stratification. When DLBCL patients with low IPI scores were divided into two groups per NONHSAG026900 expression values, the HR (low vs. high value group) was 2.010 (95\% CI: 1.253-3.222; Figure 5C). Also, 5-year OS rates $(55.27 \%, n=138)$ in the low value group had significantly worse prognosis than those $(79.83 \%, n=82)$ in the high value group $(P=0.003$; Figure 5C). We obtained similar results from the high IPI score group, with a significant HR value (1.835, low vs. high value group; 95\% CI: 0.973-3.461) and 5-year OS rates $(34.16 \%$ vs. $51.95 \% ; P=0.006$; Figure 5D). Overall, we could identify $67.96 \%$ of all subjects as especially short survival DLBCL patients by evaluating individuals who had low or high IPI scores along with a low-value expression profile (the green line) of NONHSAG026900. Taken together, all of these results suggested that NONHSAG026900 could add to the prognostic power of the IPI as an independent factor. 


\section{Prediction of NONHSAG026900 function}

We had identified NONHSAG026900 expression patterns as having diagnostic and prognostic value in DLBCL and therefore wanted to know its biological function. To identify the function of NONHSAG026900 and its potential role in DLBCL pathogenesis we utilized the gene expression profiles from 11 DLBCL samples to develop a coding-non-coding co-expression network. The co-expression network was composed of 856 lncRNA genes and 3,998 coding genes with 9,518 noncoding-noncoding edges, 113,085 coding-coding edges and 25,641 noncoding-coding edges. We predicted NONHSAG026900 functions via two different methods (a module-based analysis and a hub-based analysis). Primarily, a total of 48 module-based subnetworks were produced from the co-expression network by using an MCL algorithm. There were 18 of these modules with at least one enriched GO term. Through parsing the coexpression network into different hub-based subnetworks, we observed 457 IncRNA centered subnetworks with GO term enrichment. Our results demonstrated that NONHSAG026900 was classified into the same module with 134 noncoding genes and 1,174 coding genes.

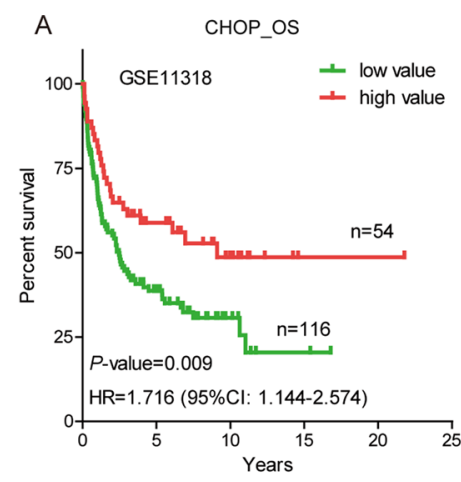

B

CHOP_OS
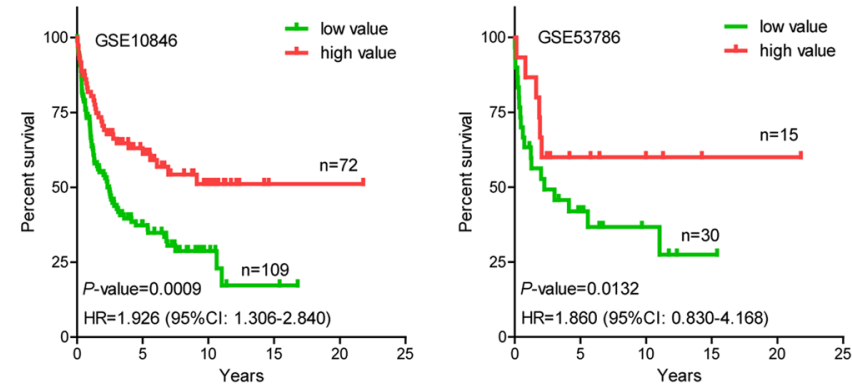

C

R-CHOP_OS
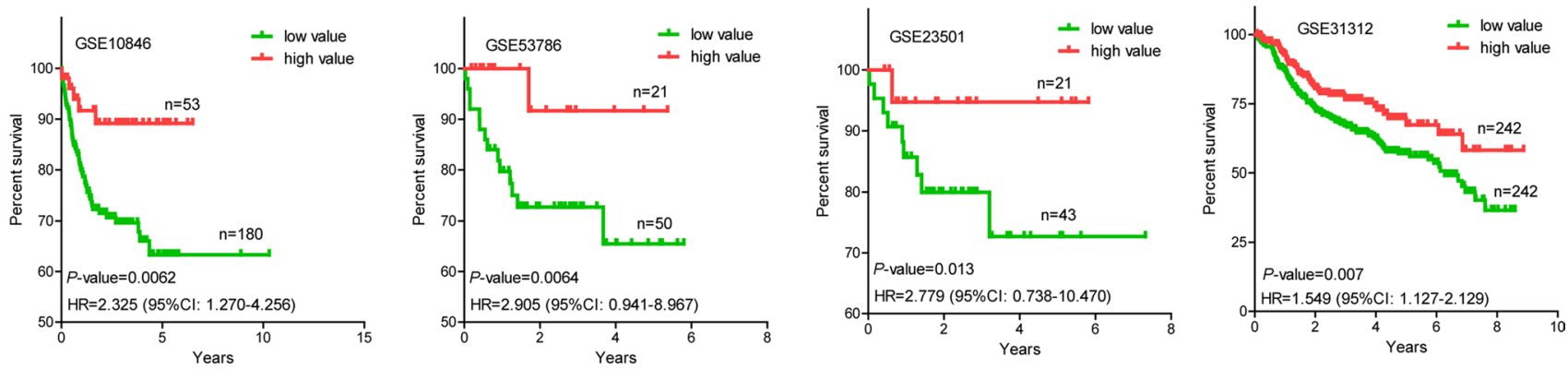

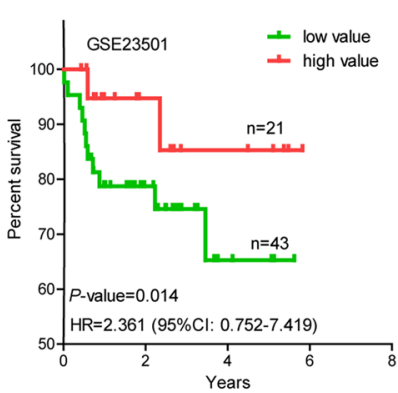

R-CHOP_PFS

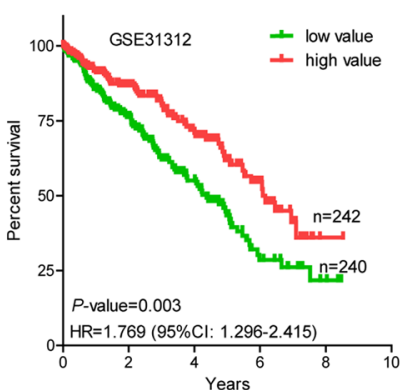

E

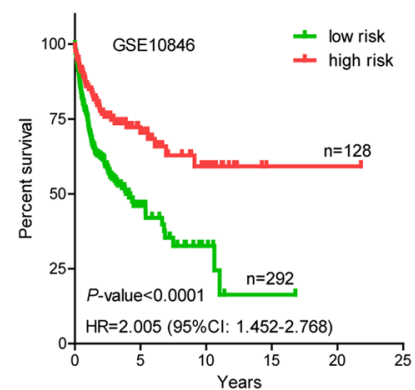

CHOP/R-CHOP_OS

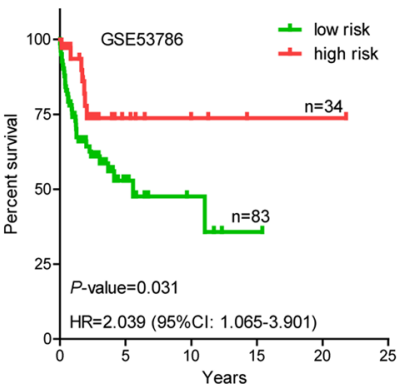

Figure 4: Overall survival (OS) or progression-free survival (PFS) analysis of DLBCL patients with CHOP/R-CHOP regimens in the training and validated cohorts. (A) A Kaplan-Meier survival curve analysis showed that patients with higher expression of NONHSAG026900 showed increased overall survival (OS) compared with lower expression of NONHSAG026900 in GSE11318. The $P$-value was calculated by a log-rank test. The Kaplan-Meier plots were used to visualized the OS or PFS probabilities for the high value versus low value group of patients based on the threshold value of mean expression. (B) OS analysis by Kaplan-Meier curves for GSE10846 ( $n=181)$ and GSE53486 $(n=45)$ patients with CHOP regimen; (C) OS analysis by Kaplan-Meier curves for GSE10846 $(n=233)$, GSE53786 $(n=71)$, GSE23501 $(n=64)$ and GSE31312 $(n=484)$ patients with R-CHOP regimen; (D) PFS analysis by KaplanMeier curves for GSE23501 $(n=64)$ and GSE31312 $(n=482)$ patients with R-CHOP regimen; (E) OS analysis by Kaplan-Meier curves for all GSE10846 $(n=420)$ and GSE53486 $(n=117)$ patients with CHOP/R-CHOP regimen. The tick marks on the Kaplan-Meier curves represented the censored subjects. The $P$-value was calculated by the two-sided log-rank test. 
Table 2: Univariate and multivariate analyses of prognostic variables in patients with DLBCL

\begin{tabular}{|c|c|c|c|c|c|c|}
\hline \multirow{2}{*}{ Variables } & \multicolumn{3}{|c|}{ Univariate analysis } & \multicolumn{3}{|c|}{ Multivariate analysis } \\
\hline & HR & $95 \%$ CI & $P$-value & HR & $95 \% \mathrm{CI}$ & $P$-value \\
\hline \multicolumn{7}{|l|}{ GSE11318 } \\
\hline NONHSAG026900 (low vs. high) & 1.716 & $1.144-2.574$ & $0.009^{*}$ & 0.928 & $0.809-1.064$ & $0.028 *$ \\
\hline Gender (Female vs. Male) & 0.997 & $0.673-1.478$ & 0.920 & & & \\
\hline Genotype (GCB vs. Non-GCB) & 0.475 & $0.321-0.704$ & $0.002 *$ & 1.073 & $0.778-1.478$ & $0.038^{*}$ \\
\hline IPI scores (0-2 vs. 3-5) & 0.224 & $0.128-0.391$ & $<0.0001 *$ & 1.718 & $1.401-2.107$ & $0.000^{*}$ \\
\hline \multicolumn{7}{|l|}{ GSE23501 } \\
\hline NONHSAG026900 (low vs. high) & 2.779 & $0.738-10.470$ & $0.013^{*}$ & 0.660 & $0.186-2.343$ & $0.025^{*}$ \\
\hline Gender (Female vs. Male) & 1.211 & $0.356-4.126$ & 0.759 & & & \\
\hline Genotype (GCB vs. Non-GCB) & 0.288 & $0.095-0.876$ & $0.028 *$ & 2.944 & $0.828-10.469$ & 0.051 \\
\hline IPI scores (0-2 vs. 3-5) & 1.498 & $0.466-0.482$ & 0.497 & & & \\
\hline \multicolumn{7}{|l|}{ GSE53786 } \\
\hline NONHSAG026900 (low vs. high) & 2.039 & $1.065-3.901$ & $0.031^{*}$ & 0.967 & $0.714-1.311$ & $0.031^{*}$ \\
\hline Gender (Female vs. Male) & 0.994 & $0.543-1.817$ & 0.788 & & & \\
\hline Genotype (GCB vs. Non-GCB) & 0.463 & $0.247-0.868$ & $0.016^{*}$ & 1.752 & $0.589-5.210$ & 0.122 \\
\hline IPI scores (0-2 vs. 3-5) & 0.274 & $0.149-0.504$ & $<0.0001 *$ & 2.276 & $1.627-3.184$ & $0.000^{*}$ \\
\hline \multicolumn{7}{|l|}{ GSE10846 } \\
\hline NONHSAG026900 (low vs. high) & 2.005 & $1.452-2.768$ & $<0.0001 *$ & 1.021 & $0.625-1.670$ & $0.010^{*}$ \\
\hline Gender (Female vs. Male) & 0.918 & $0.608-1.386$ & 0.954 & & & \\
\hline Genotype (GCB vs. Non-GCB) & 0.497 & $0.333-0.741$ & $0.000^{*}$ & 2.123 & $1.341-3.363$ & $0.001 *$ \\
\hline IPI scores (0-2 vs. $3-5)$ & 0.270 & $0.178-0.411$ & $0.000^{*}$ & 2.679 & $1.880-3.816$ & $0.000^{*}$ \\
\hline \multicolumn{7}{|l|}{ GSE31312 } \\
\hline NONHSAG026900 (low vs. high) & 1.549 & $1.127-2.129$ & $0.007^{*}$ & 1.549 & $1.127-2.129$ & $0.007 *$ \\
\hline Gender (Female vs. Male) & 1.010 & $0.745-1.371$ & 0.948 & & & \\
\hline Genotype (GCB vs. Non-GCB) & 0.915 & $0.675-1.241$ & 0.567 & & & \\
\hline IPI scores (0-2 vs. $3-5)$ & 1.324 & $0.980-1.791$ & 0.068 & & & \\
\hline
\end{tabular}

Abbreviations: HR: hazard ratio; CI: confidence interval * Statistically significant $(P<0.05)$.

Because we could infer that genes in the same co-expressed module had similar functions, the role of NONHSAG026900 might be closely associated with those 1,174 coding genes. Therefore, we concluded that the functions of NONHSAG026900 were probably involved with mitosis and cell cycle progression (Supplementary Figure 1, and Supplementary Table 2). Subsequently, we re-analyzed the potential functions of NONHSAG026900 with hub-based analysis, and we discovered that it was surrounded by 55 protein-coding genes (Supplementary Figure 2). In line with the predictions of the module-based method, NONHSAG026900 was predicted to suppress mitotic cell cycle (Supplementary Table 3).

To validate the predicted function of NONHSAG026900 in cell proliferation was involved in its prognostic power, we compared the 55 proteincoding genes around NONHSAG026900 correlated with cell cycle regulation and 32 genes reported to be associated with prognosis of DLBCL. We discovered three common protein-coding genes (MYBL1, MME and LRMP) in the two groups (Figure 6A). Next, we investigated whether there was any interaction between MYBL1, MME, LRMP and NONHSAG026900. The expression of NONHSAG026900 was positively correlated with the mRNA expression values of MYBL1, MME and LRMP in the DLBCL tissues from GSE12453 (Figure 6A, Pearson $r>0.9, P<0.001$ ). Moreover, the expressions of these three protein-coding genes were also significantly down-regulated in DLBCL samples when compared with normal samples $(P<0.05$, Figure $6 \mathrm{~B})$, which agreed with the results from NONHSAG026900 outlined above. Meanwhile, we analyzed the expressions of MYBL1, MME and LRMP between the GCB and nonGCB subgroups in patients from GSE11318. The three genes were significantly down-regulated in the non-GCB compared to the GCB subgroup $(P<0.001$, Figure 6C), which was also in line with the NONHSAG026900 expression distribution. Interestingly, some studies 
reported that these three genes could be prognostic biomarkers for DLBCL because they stimulate cell proliferation and differentiation [23, 25-30]. Taken together, our results suggested that the prognostic power of NONHSAG026900 was related to its predicted role in cell proliferation regulation like the three protein-coding biomarkers in patients with DLCBL.

\section{DISCUSSION}

DLBCL is one of the most pathologically heterogeneous cancers, and a large number of patients relapse within 2-3 years after primary chemotherapy [31]. Therefore, it is significantly important to discover an effective prognostic marker to apply into clinical practice.
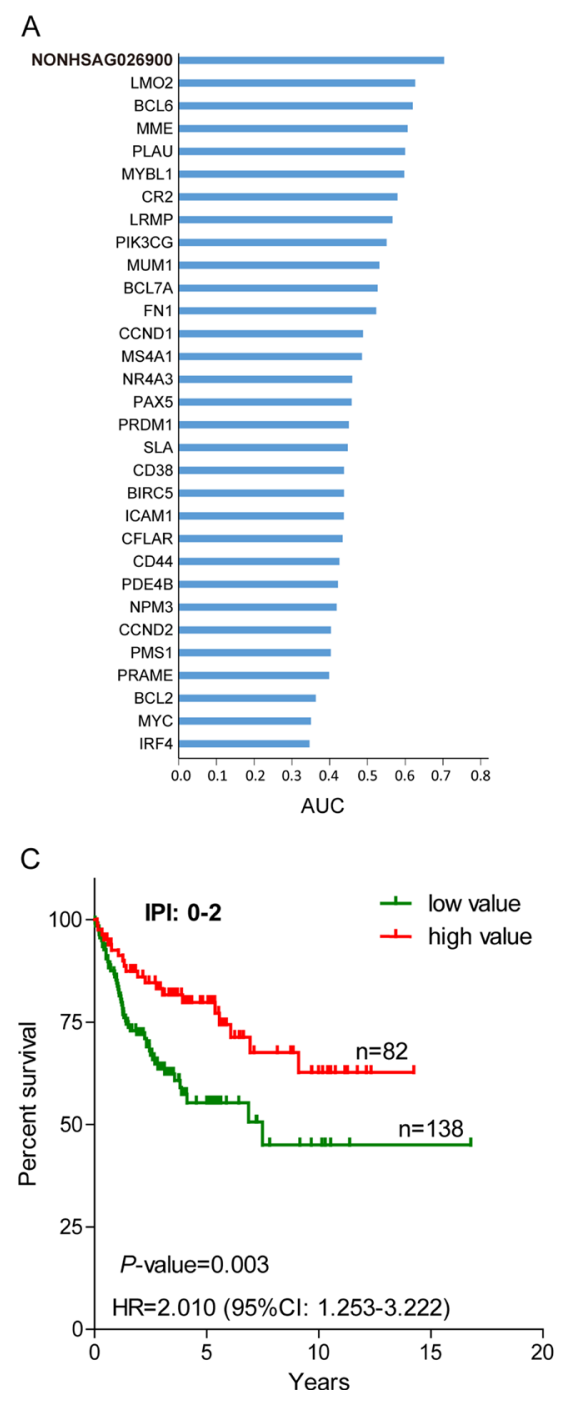

Some research revealed that many lncRNAs were involved in the process of tumor, including cancer cell proliferation, adhesion, migration, and cell cycle [32-34]. And the correlation between IncRNAs and cancer significance has shown that some lncRNAs could be utilized as biomarkers for prognosis or diagnosis [35-37]. Meng Zhou etc. identified a 17-lncRNA signature to classify subtype and predict prognosis in 1,118 patients with DLBCL [38]; Yuling Yan etc. reported a lncRNA HOTAIR could predict a poor prognosis, which promotes cell proliferation and is correlated with tumor size and IPI [39]; Wei Peng etc. demonstrated that the IncRNA LUNAR1 and PEG10 as indicators of poor survival rate may play a key oncogene role promoting cell proliferation for DLBCL patients $[40,41]$. Despite the progress has been made in the

B
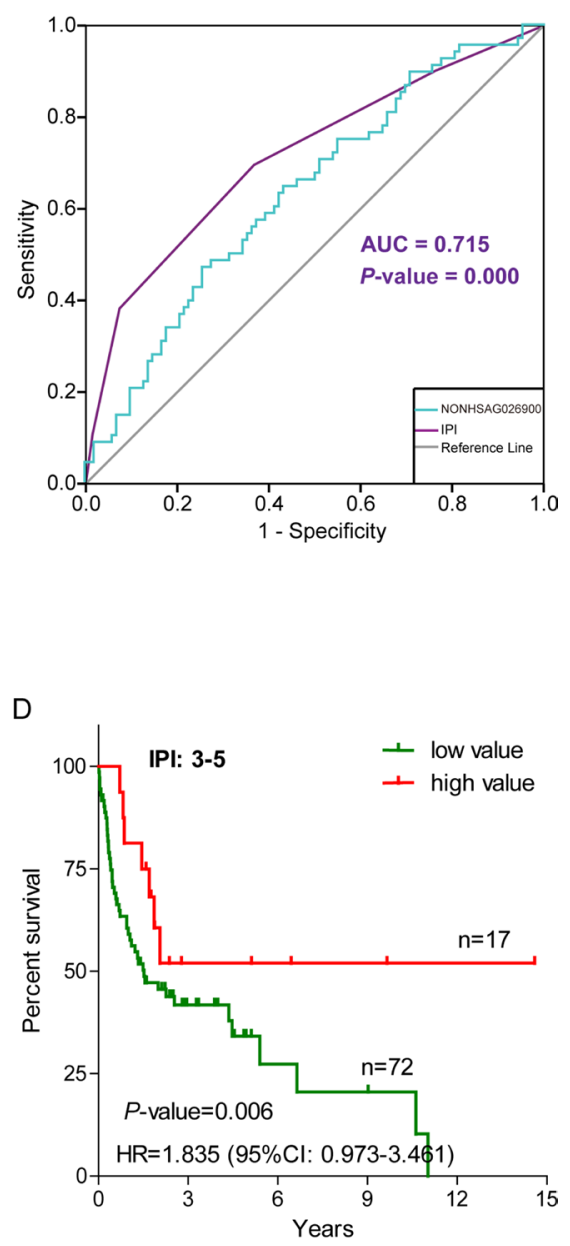

Figure 5: The prognostic power of NONHSAG026900 compared with other predictive biomarkers and the International Prognostic Index (IPI). (A) A bar plot was used to show that the area under curve (AUC) value of NONHSAG026900 was greater than 30 other prognostic biomarkers reported previously; (B) Receiver operating characteristic (ROC) curve analysis was employed to compare the predictive power between NONHSAG026900 (AUC $=0.703, P=0.003$ ) and IPI (AUC $=0.715, P=0.000)$; (C and D) The KaplanMeier curves showed overall survival for groups of patients with low IPI scores (0-2) and high IPI scores (3-5) after classification into the low value group or high value group on the basis of cutoff values with the mean. According to log-likelihood estimates, $P=0.003(\mathrm{C})$ and $P=0.006$ (D) for the model based on a continuous variable applied to the low and high value groups shown in the figure, respectively. 
prognostic biomarkers of lncRNAs in DLBCL patients, further research needs to be done to discover more precise forecast genes.

In our study, we re-analyzed 7 microarray datasets to examine the pathogenesis of DLBCL. And we found the lncRNA NONHSAG026900 was significantly lower expression in DLBCL cells compared with the five differentiated stages of normal B cells $(P<0.05)$. We also discovered that the expression of NONHSAG026900 was significantly higher in GCB-DLBCL patients than non-GCB-DLBCL patients $(P=0.000)$. Because patients with GCB-DLBCL have a more favorable outcome than those with non-GCB-DLBCL [42], we inferred that NONHSAG026900 might be used as a biomarker to predict the prognosis of DLBCL patients. Subsequently, we confirmed our discovery in patients with CHOP or R-CHOP regimens from another four GEO datasets (GSE10846, GSE53786, GSE23501, and GSE31312). Kaplan-Meier analysis suggested that patients with low expression of NONHSAG026900 had significantly higher risk and shorter 5-year OS or PFS ratios than those from the high value group. In addition, we determined the correlation between NONHSAG026900 and IPI, and found that the NONHSAG026900 could add to the predictive power of IPI as an independent biomarker from multivariate Cox regression analysis. To the best of our knowledge, this is the first report that showed that NONHSAG026900 was a favorable predictor of survival in a large group of DLBCL patients.

The cell cycle is a critical factor that controls cell division during cancer development. MYBL1, MME and LRMP, as key regulators of the cell cycle, have been identified as potential therapeutic targets for cancer. MYBL1, which is located in chromosome region $8 \mathrm{q} 22$, could be involved in recurrent translocations in malignant lymphoma [28]. MYBL1 is highly expressed in Burkitt's lymphoma cells, some chronic lymphocytic leukemia, and a small subset of human neoplastic B-cells and stimulates the proliferation and differentiation as a member of the Myb oncogene family of transcription factors [29, 43, 44]. It is specifically induced in proliferating centroblasts and acts as a specific marker
A

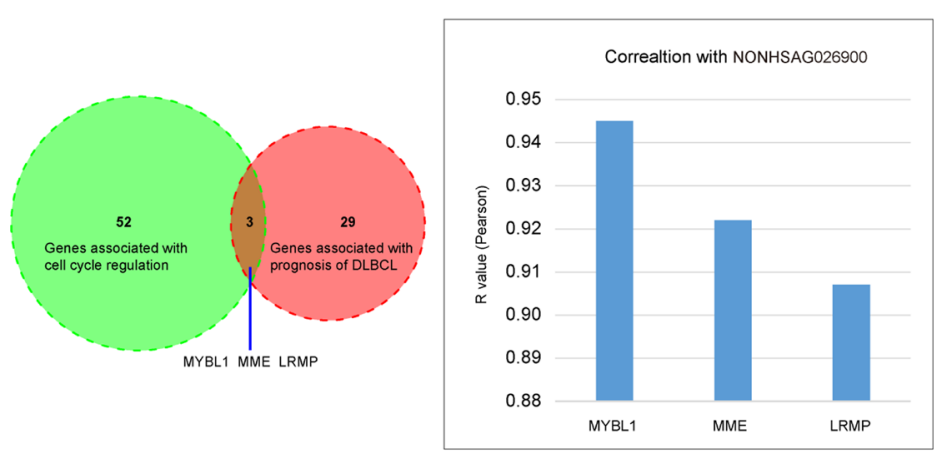

B

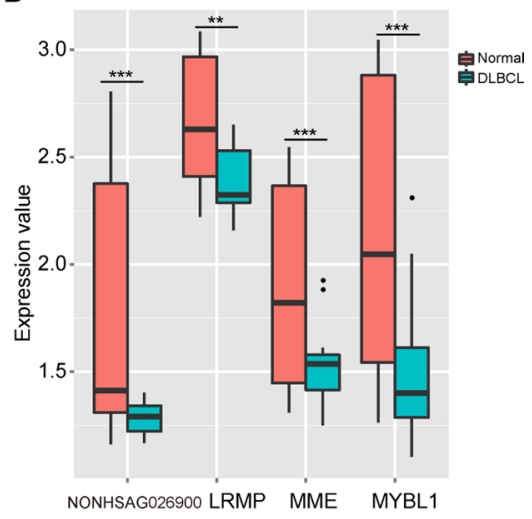

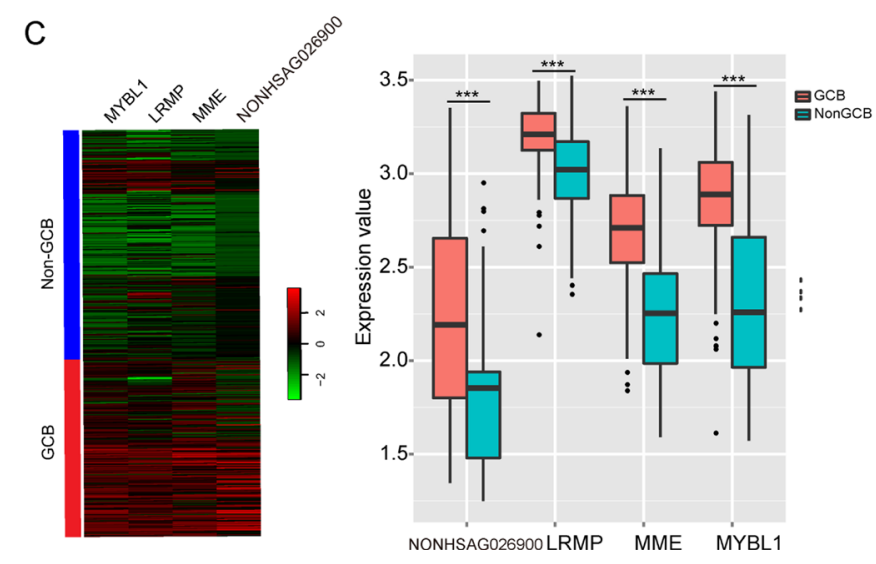

Figure 6: LncRNA NONHSAG026900 functional prediction and validation. (A) A Venn diagram showed the overlap between the genes associated with cell cycle regulation and the genes correlated with prognosis of DLBCL, and identified the three overlapping genes; the bar plot showed the correlation between the three overlapping genes and NONHSAG026900 (Pearson $r>0.9, P<0.001)$; (B) Box plots showed that the three protein coding-genes (MYBL1, MME and LRMP) were significantly down-regulated in DLBCL compared to normal tissues in patients from GSE12453 ( $P<0.001$, $t$-test method), in addition to NONHSAG026900; (C) Microarray gene expression heatmap of the four genes (MYBL1, MME, LRMP and NONHSAG026900) between GCB and non-GCB subgroups in GSE11318. The expression values of the four genes were significantly higher in the GCB than non-GCB subgroup $(P<0.001, t$-test method) in the boxplot on the right. 
for the proliferation of centroblasts [30]. However, Golay etc. discovered that MYBL1 mRNA was very weak or negative in DLBCL B-cell lines compared with normal tonsillar buoyant B cells [44], which demonstrated that the similar change trend with our analysis in Figure 6B. $\mathrm{MME}$, is a proliferation blocker, can cleave signal peptides at the cell surface to affect cell proliferation and differentiation, and acts as an acute lymphocytic leukemia antigen $[26,27]$. In our research, the expression of MME was significantly down-regulated in the nonGCB compared to the GCB subgroup. Therefore, improved expression of MME was associated with a favorable outcome in DLBCL patients. The lymphoidrestricted membrane protein (LRMP), is an endoplasmic reticulum-associated protein. We found higher mRNA levels of LRMP in the GCB- compared to the non-GCBsubtype of DLBCL, which demonstrated that it predicted and supported the aggressive behavior of the non-GCB subtype of DLBCL $[23,25]$. In this study, we discovered that NONHSAG026900 was clustered with these three protein-coding genes into one functional module for cell cycle regulation by gene co-expression network analysis. NONHSAG026900 was significantly down regulated in DLBCL samples, especially in the non-GCB subtype, which suggested that its presence could inhibit cell cycle activity to restrain tumor growth and thereby predict the prognosis of patients with DLBCL.

We agreed that the biological validation is in some way weak in this study. We understand that it is better to reveal the potential lncRNA transcriptional mechanism by the examination of the methylated sites in corresponding cancer cells and the expression of lncRNA NONHSAG026900. However, in the present study, we mainly focused on exploring an analysis method with bioinformatics tools to reveal the mechanism of lncRNA NONHSAG026900 with lower expression in DLBCL patients. And the further efforts in the next study will be paid to achieve the corresponding experiment above and validate the discoveries about the expression and function of this lncRNA with modern empirical method of molecular biology.

Generally, our results demonstrated that decreased NONHSAG026900 expression was observed frequently in DLBCL and could be identified as a novel biomarker for diagnosis and an independent factor for predicting prognosis of patients with DLBCL. This suggested that NONHSAG026900 might enhance tumor suppression as an indicator of favorable survival ratio, and function as a positive prognostic factor for patients with DLBCL. Moreover, a deeper understanding of the mechanisms of NONHSAG026900 in DLBCL will promote the development of NONHSAG026900-directed diagnostic, prognostic and therapeutic agents against this malignancy.

\section{MATERIALS AND METHODS}

\section{Microarray data and patient information}

We obtained published Affymetrix platform HG-U133A Plus 2.0 microarray data sets from the Gene Expression Omnibus (GEO) database (accession numbers: GSE12453, GSE56315, GSE11318, GSE23501, GSE53786, GSE10846, and GSE31312). The microarray data assessed the gene expression profiles of 36 samples consisting of 5 naïve B-cell samples, 5 centroblast samples, 5 centrocyte samples, 5 memory B cell samples, 5 plasma cell samples, and 11 DLBCL samples in GSE12453. Similarly, there were 6 naïve B-cell samples, 7 centroblast samples, 7 centrocyte samples, 6 memory B cell samples, 7 plasma cell samples, and 74 DLBCL samples in the validated cohort GSE56315. In the other five independent cohorts, a total of 170, 420, 117, 69, and 484 patients were from GSE11318, GSE10846, GSE53786, GSE23501, and GSE31312, respectively. The number of patients with CHOP, R-CHOP like, or other regimens were 164, 0 and 6 in GSE11318. GSE10846 was composed of 181 patients with CHOP-like regimen, 233 patients with R-CHOP regimen, and 6 patients with other regimens, while the number of patients with $\mathrm{CHOP}$, R-CHOP like or other regimens was 45,71 and 1 in GSE53786, and 1, 64, and 4 in GSE23501, respectively. However, 484 patients with R-CHOP like regimen only were selected from the GSE31312 cohort.

\section{Repurposing microarray data}

We quantified the expression levels of messenger RNAs and long non-coding RNAs by the re-annotation of Affymetrix microarray probes using the Non-coding RNA Function Annotation Server (ncFANs) [45]. We submitted 7 microarray datasets with a CEL format to ncFANs and acquired lncRNA and protein-coding gene expression values ( $\log 2$-transformed) using R. Meanwhile, we used the Limma statistical package to implement the Robust Multichip Average (RMA) analysis, Student's $t$-test, one-way ANOVA (paired with an F test) and Benjamini Hochberg $(\mathrm{BH})$ false discovery rate (FDR) correction. Therefore, we considered genes with a Fold-Change $>2$ and BH FDR-adjusted $p$-values $<0.05$ as those with different expressions.

\section{Functional enrichment of protein-coding genes}

We utilized the DAVID Bioinformatics Tool [46] to perform the functional enrichment of target protein-coding genes. We identified the biological processes related to protein-coding genes by conducting gene ontology analysis with this tool. In our study, we defined $p$-values 
$<0.05$ as a significance threshold. Finally, the Enrichment Map plugin [47] for Cytoscape [48] was used to visualize the biological process organization.

\section{Functional analysis of IncRNAs}

We utilized gene expression data of 36 tissue samples from GSE12453 to produce a co-expression network including non-coding and protein-coding genes. Then we chose both protein-coding and non-coding genes with different expressions to construct a co-expression network. We evaluated the relationship of each gene pair (coding-coding, coding-lncRNA, and lncRNA-lncRNA gene pairs) with a Pearson's correlation coefficient or a Spearman Rank correlation coefficient. We utilized Fisher's asymptotic test to calculate the $p$-values of the correction coefficients for each gene pair, and then adjusted this with a Bonferroni multiple testing correction. Next, we developed the coding-lncRNA co-expression network by selecting co-expression gene pairs with $p$-values $<0.01$. Based on the coding-lncRNA co-expression network, we predicted the functions of lncRNAs by module-based and hub-based methods embedded in ncFANs. We identified modules of co-expressed genes in the coding-lncRNA co-expression network by the Markov cluster algorithm (MCL) in the module based method. We further analyzed the protein-coding genes in the same module as the lncRNAs by gene ontology analysis in order to predict the functions of lncRNAs in this module. Subsequently, the co-expression network was parsed into several subnetworks with the hub-based method, which consisted of some protein coding genes around a central lncRNA. We inferred the function of the corresponding lncRNA by the functional enrichment of these connected protein-coding genes. In our analysis, we only reserved the function enrichments with $p$-values $<0.01$.

\section{Statistical analysis}

Pearson Chi-square tests and the student's $t$ test analysis of variance were utilized to analyze statistical differences in demographic and clinical characteristics. The paired student-test compared distributive differences of NONHSAG026900 expression between germinal center B-cell like (GCB) and non-GCB subgroups. Receiver-operating characteristic (ROC) curve analysis was used to visualize the specificity and sensitivity of the biomarker NONHSAG026900 in predicting prognosis of patients with DLBCL. The performance was quantified by the area under the ROC curve. The correlation between NONHSAG026900 expression and overall survival (OS) or progression-free survival (PFS) of patients was assessed by univariate Cox regression analysis. Survival differences between low and high value groups in each set were evaluated by the Kaplan-Meier estimate, and compared by the log-rank test. To explore whether the predictive power of NONHSAG026900 was independent of IPI scores, multivariate Cox regression analysis and data stratification analysis were conducted in our study. Statistical analysis was performed with SPSS 13.0, and presented with GraphPad Prism 5.0 and R3.2.2 software. Results were considered statistically significant at $P<0.05$.

\section{Authors' contributions}

S.Z. performed the biostatistics analysis and constituted the prediction model, S.F. re-annotated all the collected probes, Y.L. collected GEO data samples. Y.Z., L.W. and R. X. conceived and designed the experiments.

\section{ACKNOWLEDGMENTS AND FUNDING}

We thank Dr. Peiqing Sun (Department of Cancer Biology, School of Medicine, Wake Forest University, USA) for assistance in writing the paper and provided important advices. This work was supported by National Natural Science Foundation of China [31371320, 31401119, 61472397, and 31501066].

\section{CONFLICTS OF INTEREST}

The authors declare that they have no competing interests.

\section{REFERENCES}

1. Roschewski M, Staudt LM, Wilson WH. Diffuse large B-cell lymphoma [mdash] treatment approaches in the molecular era. Nature Reviews Clinical Oncology. 2014; 11:12-23.

2. Feugier P. Long-Term Results of the R-CHOP Study in the Treatment of Elderly Patients With Diffuse Large B-Cell Lymphoma: A Study by the Groupe d'Etude des Lymphomes de l'Adulte. Journal of Clinical Oncology. 2005; 23:4117-4126.

3. Fu K, Weisenburger DD, Choi WWL, Perry KD, Smith LM, Shi X, Hans CP, Greiner TC, Bierman PJ, Bociek RG, Armitage JO, Chan WC, Vose JM. Addition of Rituximab to Standard Chemotherapy Improves the Survival of Both the Germinal Center B-Cell-Like and Non-Germinal Center B-Cell-Like Subtypes of Diffuse Large B-Cell Lymphoma. Journal of Clinical Oncology. 2008; 26:4587-4594.

4. Vaidya R, Witzig T. Prognostic factors for diffuse large B-cell lymphoma in the R (X) CHOP era. Annals of oncology. 2014:mdu109.

5. Friedberg JW. Relapsed/refractory diffuse large B-cell lymphoma. ASH Education Program Book. 2011; 2011:498-505.

6. Hu Y, Ding N, Jin X, Feng L, Ping L, Song Y, Zhu J. Genetic polymorphisms of STAT3 correlated with prognosis 
in diffuse large B-cell lymphoma patients treated with rituximab. Cancer cell international. 2014; 14:1-6.

7. Habermann TM, Wang SS, Maurer MJ, Morton LM, Lynch CF, Ansell SM, Hartge P, Severson RK, Rothman N, Davis S, Geyer SM, Cozen W, Chanock SJ, et al. Host immune gene polymorphisms in combination with clinical and demographic factors predict late survival in diffuse large B-cell lymphoma patients in the pre-rituximab era. Blood. 2008; 112:2694-2702.

8. Ponting CP, Belgard TG. Transcribed dark matter: meaning or myth? Human Molecular Genetics. 2010:ddq362.

9. Kumarswamy R, Bauters C, Volkmann I, Maury F, Fetisch J, Holzmann A, Lemesle G, de Groote P, Pinet F, Thum T. Circulating long noncoding RNA, LIPCAR, predicts survival in patients with heart failure. Circulation research. 2014; 114:1569-1575.

10. Dorn GW. LIPCAR A Mitochondrial lnc in the Noncoding RNA Chain? Circulation research. 2014; 114:1548-1550.

11. Li J, Chen Z, Tian L, Zhou C, He MY, Gao Y, Wang S, Zhou F, Shi S, Feng X. LncRNA profile study reveals a threelncRNA signature associated with the survival of patients with oesophageal squamous cell carcinoma. Gut. 2014; 63:1700-10.

12. Prensner JR, Chinnaiyan AM. The emergence of lncRNAs in cancer biology. Cancer discovery. 2011; 1:391-407.

13. Reis EM, Verjovski-Almeida S. Perspectives of long noncoding RNAs in cancer diagnostics. Frontiers in genetics. 2012; 3 .

14. Tsai MC, Spitale RC, Chang HY. Long intergenic noncoding RNAs: new links in cancer progression. Cancer research. 2011; 71:3-7.

15. Feng S, Yao J, Chen Y, Geng P, Zhang H, Ma X, Zhao J, $\mathrm{Yu}$ X. Expression and Functional Role of ReprogrammingRelated Long Noncoding RNA (lincRNA-ROR) in Glioma. Journal of Molecular Neuroscience. 2015:1-8.

16. Peng W, Wu J, Feng J. LincRNA-p21 predicts favorable clinical outcome and impairs tumorigenesis in diffuse large B cell lymphoma patients treated with R-CHOP chemotherapy. Clinical and experimental medicine. 2015:1-8.

17. Kundaje A, Meuleman W, Ernst J, Bilenky M, Yen A, Heravi-Moussavi A, Kheradpour P, Zhang Z, Wang J, Ziller MJ. Integrative analysis of 111 reference human epigenomes. Nature. 2015; 518:317-330.

18. Fu K, Weisenburger DD, Choi WW, Perry KD, Smith LM, Shi X, Hans CP, Greiner TC, Bierman PJ, Bociek RG. Addition of rituximab to standard chemotherapy improves the survival of both the germinal center B-cell-like and non-germinal center B-cell-like subtypes of diffuse large B-cell lymphoma. Journal of Clinical Oncology. 2008; 26:4587-4594.

19. Lossos IS, Czerwinski DK, Alizadeh AA, Wechser MA, Tibshirani R, Botstein D, Levy R. Prediction of survival in diffuse large-B-cell lymphoma based on the expression of six genes. New England Journal of Medicine. 2004; 350:1828-1837.
20. Mitsuhashi K, Masuda A, Wang YH, Shiseki M, Motoji T. Prognostic significance of PRAME expression based on immunohistochemistry for diffuse large B-cell lymphoma patients treated with R-CHOP therapy. International journal of hematology. 2014; 100:88-95.

21. Rydström K, Linderoth J, Nyman H, Ehinger M, Joost P, Bendahl PO, Leppä S, Jerkeman M. CD40 is a potential marker of favorable prognosis in patients with diffuse large B-cell lymphoma treated with immunochemotherapy. Leukemia \& lymphoma. 2010; 51:1643-1648.

22. Suzuki Y, Yoshida T, Wang G, Togano T, Miyamoto S, Miyazaki K, Iwabuchi K, Nakayama M, Horie R, Niitsu N. Association of CD20 levels with clinicopathological parameters and its prognostic significance for patients with DLBCL. Annals of hematology. 2012; 91:997-1005.

23. Blenk S, Engelmann J, Weniger M, Schultz J, Dittrich M, Rosenwald A, Müller-Hermelink HK, Müller T, Dandekar T. Germinal center B cell-like (GCB) and activated B cell-like (ABC) type of diffuse large B cell lymphoma (DLBCL): analysis of molecular predictors, signatures, cell cycle state and patient survival. Cancer informatics. 2007; 3:399.

24. Li J, Hou J, Li L, Wang Y. Immunohistochemical subtypes of diffuse large B-cell lymphoma in the head and neck region. Genetics and molecular research: GMR. 2015; 14:3889.

25. Tedoldi S, Paterson J, Cordell J, Tan SY, Jones M, Manek S, Dei Tos A, Roberton H, Masir N, Natkunam Y. Jaw1/LRMP, a germinal centre-associated marker for the immunohistological study of B-cell lymphomas. The Journal of pathology. 2006; 209:454-463.

26. Thompson RC, Herscovitch M, Zhao I, Ford TJ, Gilmore TD. NF-kappaB down-regulates expression of the B-lymphoma marker CD10 through a miR-155/PU.1 pathway. Journal of Biological Chemistry. 2011; 286:1675-1682.

27. Sumitomo M, Shen R, Nanus DM. Involvement of neutral endopeptidase in neoplastic progression. Biochimica et Biophysica Acta (BBA)-Proteins and Proteomics. 2005; 1751:52-59.

28. Barletta C, Druck T, LaForgia S, Calabretta B, Drabkin H, Patterson D, Croce CM, Huebner K. Chromosome locations of the MYB related genes, AMYB, BMYB. Cancer research. 1991; 51:3821-3824.

29. Golay J, Basilico L, Loffarelli L, Songia S, Broccoli V, Introna M. Regulation of hematopoietic cell proliferation and differentiation by themyb oncogene family of transcription factors. International Journal of Clinical and Laboratory Research. 1996; 26:24-32.

30. Golay J, Broccoli V, Lamorte G, Bifulco C, Parravicini C, Pizzey A, Thomas NSB, Delia D, Ferrauti P, Vitolo D. The A-Myb transcription factor is a marker of centroblasts in vivo. The Journal of Immunology. 1998; 160:2786-2793.

31. Seymour JF, Talpaz M, Cabanillas F, Wetzler M, Kurzrock R. Serum interleukin-6 levels correlate with prognosis in diffuse large-cell lymphoma. Journal of clinical oncology. 1995; 13:575-582. 
32. Peng W, Gao W, Feng J. Long noncoding RNA HULC is a novel biomarker of poor prognosis in patients with pancreatic cancer. Medical Oncology. 2014; 31:1-7.

33. Peng W, Wu G, Fan H, Wu J, Feng J. Long noncoding RNA SPRY4-IT1 predicts poor patient prognosis and promotes tumorigenesis in gastric cancer. Tumor Biology. 2015:1-8.

34. Peng W, Fan $\mathrm{H}, \mathrm{Wu}$ G, Wu J, Feng J. Upregulation of long noncoding RNA PEG10 associates with poor prognosis in diffuse large B cell lymphoma with facilitating tumorigenicity. Clinical and experimental medicine. 2015:1-6.

35. Fernando TR, Rodriguez-Malave NI, Waters EV, Yan W, Casero D, Basso G, Pigazzi M, Rao DS. LncRNA Expression Discriminates Karyotype and Predicts Survival in B-Lymphoblastic Leukemia. Molecular Cancer Research. 2015; 13:839-851.

36. Sehgal L, Mathur R, Braun FK, Wise JF, Berkova Z, Neelapu S, Kwak LW, Samaniego F. FAS-antisense 1 lncRNA and production of soluble versus membrane Fas in B-cell lymphoma. Leukemia. 2014; 28:2376-2387.

37. Wang J, Pan Y, Wu J, Zhang C, Huang Y, Zhao R, Cheng G, Liu J, Qin C, Shao P, Hua L, Wang Z. The Association between Abnormal Long Noncoding RNA MALAT-1 Expression and Cancer Lymph Node Metastasis: A Meta-Analysis. BioMed research international. 2016; 2016:1823482.

38. Zhou M, Zhao H, Xu W, Bao S, Cheng L, Sun J. Discovery and validation of immune-associated long non-coding RNA biomarkers associated with clinically molecular subtype and prognosis in diffuse large B cell lymphoma. Molecular Cancer. 2017; 16:16.

39. Yan Y, Han J, Li Z, Yang H, Sui Y, Wang M. Elevated RNA expression of long noncoding HOTAIR promotes cell proliferation and predicts a poor prognosis in patients with diffuse large B cell lymphoma. Molecular Medicine Reports. 2016; 13:5125-5131.

40. Peng W, Fan H, Wu G, Wu J, Feng J. Upregulation of long noncoding RNA PEG10 associates with poor prognosis in diffuse large B cell lymphoma with facilitating tumorigenicity. Clinical And Experimental Medicine. 2016; 16:177-182.
41. Peng W, Feng J. Long noncoding RNA LUNAR1 associates with cell proliferation and predicts a poor prognosis in diffuse large B-cell lymphoma. Biomedicine \& Pharmacotherapy. 2016; 77:65-71.

42. Hans CP, Weisenburger DD, Greiner TC, Gascoyne RD, Delabie J, Ott G, Müller-Hermelink HK, Campo E, Braziel RM, Jaffe ES. Confirmation of the molecular classification of diffuse large B-cell lymphoma by immunohistochemistry using a tissue microarray. Blood. 2004; 103:275-282.

43. Lian L, Li X, Zhao C, Han B, Qu L, Song J, Liu C, Yang N. Chicken gga-miR-181a targets MYBL1 and shows an inhibitory effect on proliferation of Marek's disease virustransformed lymphoid cell line. Poultry Science. 2015; 94:2616-2621.

44. Golay J, Luppi M, Songia S, Palvarini C, Lombardi L, Aiello A, Delia D, Lam K, Crawford D, Biondi A. Expression of A-myb, but not c-myb and B-myb, is restricted to Burkitt's lymphoma, sIg+ B-acute lymphoblastic leukemia, and a subset of chronic lymphocytic leukemias. Blood. 1996; 87:1900-1911.

45. Liao Q, Xiao H, Bu D, Xie C, Miao R, Luo H, Zhao G, Yu K, Zhao H, Skogerbø G. ncFANs: a web server for functional annotation of long non-coding RNAs. Nucleic acids research. 2011; 39:W118-W124.

46. Huang DW, Sherman BT, Lempicki RA. Bioinformatics enrichment tools: paths toward the comprehensive functional analysis of large gene lists. Nucleic acids research. 2009; 37:1-13.

47. Merico D, Isserlin R, Stueker O, Emili A, Bader GD. Enrichment map: a network-based method for gene-set enrichment visualization and interpretation. PloS one. 2010; 5:e13984.

48. Shannon P, Markiel A, Ozier O, Baliga NS, Wang JT, Ramage D, Amin N, Schwikowski B, Ideker T. Cytoscape: a software environment for integrated models of biomolecular interaction networks. Genome research. 2003; 13:2498-2504. 